\title{
Thermodynamic Properties of Semiconductors with Defects
}

\author{
Vu Van Hung, Le Dai Thanh \\ Department of Physics, Hanoi National University of Education, Hanoi, Vietnam. \\ Email: bangvu57@yahoo.com
}

Received April 13 ${ }^{\text {th }}$, 2011; revised May 22 ${ }^{\text {nd }}, 2011$; accepted June $3^{\text {rd }}, 2011$.

\begin{abstract}
Thermodynamic properties of diamond cubic and zinc-blende semiconductors with point defects are considered by the statistical moment method (SMM). The thermal expansion coefficient, the specific heats at constant volume and those at constant pressure, $C_{V}$ and $C_{P}$, and the isothermal compressibility are derived analytically for semiconductors with defects. The SMM calculated thermodynamic quantities of the Si, and GaAs semiconductors with defects are in good agreement with the experimental results.
\end{abstract}

Keywords: Anharmonic Defective Semiconductor, Statistical Moment Method

\section{Introduction}

Recently, there has been a great interested in the study of bulk semiconductor, semiconductor heterostructures and nanodevices [1-4] since they provide us a wide variety of academic problems as well as the technological applications. The physical characteristics of semiconductors are determined both by the properties of the host crystal and by the presence impurities and crystalline defects. Crystal lattice defects or other impurities also modify the properties of the semiconductor and thus may make a semiconductor unsuitable for its intended applications. The point defects in semiconductors including the vacancies play an important role in many properties of material. Understanding these defects will lead to improved semiconductor devices for the technological applications.

First principles (or $a b$ initio) electronic structure computations have been performed on semiconductor compounds and the results compared with experiment [5-7]. More recently a large number of high-quanlity calculations have been performed on group-IV, III-V, and II-VI materials. Modern calculations allow the accurate relaxation of structures to their minimum energy configurations and the incorporation of temperature effects. One can also study the melting of solids and phase transitions in Si using first-principles molecular-dynamics method $[8,9]$. Such calculations are computationally expensive and, currently, simulations can only be run for periods of tens of picoseconds, which is not long enough for some of the processes of interest.

In our previous papers $[10,11]$ the statistical moment method was used to investigate the thermodynamic quantities of the elemental perfect semiconductors, taking into account the anharmonicity effects of thermal lattice vibrations. The thermal expansion coefficients, elastic moduli, specific heats at constant volume and those at constant pressure, $C_{V}$ and $C_{P}$, are derived analytically for diamond cubic semiconductors.

The purpose of the present article is to investigate the temperature dependence of the thermodynamic properties of the semiconductors with defects using the analytic statistical moment method (SMM) [12-16]. The thermodynamic quantities are derived from the Helmholtz fnee energy of semiconductors with defects.

\section{Theory}

\subsection{Atomic Displacements of Semiconductor}

To derive the temperature dependence of the thermodynamic properties of semiconductors, we use the statistical moment method. This method allows us to take into account the anharmonicity effects of thermal lattice vibrations on the thermodynamic quantities in the analytic formulations.

The essence of the SMM scheme can be summarized as follows: for simplicity, we derive the thermodynamic quantities of crystalline materials with cubic symmetry, taking into account the higher (fourth) order anharmonic contributions in the thermal lattice vibrations going be- 
yond the quasi-Hamonic $(\mathrm{QH})$ approximation. The extentions for the SMM formalism to non-cubic systems is straightforward. The basic equations for obtaining thermodynamic quantities of the given crystals are derived in a following manner: the equilibrium thermal lattice expansions are calculated by the force balance criterion and then the thermodynamic quantities are determinded for the equilibrium lattice spacings. The anharmonic contributions of the thermodynamic quantities are given explicitly in terms of the power moments of the thermal atomic displacements.

Let us first define the lattice displacements We demote $\boldsymbol{u}_{i l}$ the vector defining the displacement of the ith atom in the lth unit cell, from its equilibrium position. The potential energy of the whole crystal $U\left(\boldsymbol{u}_{i l}\right)$ is expressed in terms of the positions of all the atoms from the sites of the equilibrium lattice. We use the theory of small atomic vibrations, and expand the potential energy $U$ as a power series in the cartesian components, $\boldsymbol{u}_{i \alpha}$ of the displacement vector $\boldsymbol{u}_{i l}$ around this point.

For the evaluation of the anharmonic contributions to the free energy $\psi$, we consider a quantum system, which is influenced by supplemental forces $\alpha_{i}$ in the space of the generalized coordinates $q_{i}$. For simplicity, we only discuss monatomic systems and hereafter omit the indices $l$ on the sublattices Then, the Hamiltonian of the crystalline system is given by

$$
\widehat{H}=\widehat{H}_{0}-\sum_{i} \alpha_{i} \hat{q}_{i}
$$

where $\widehat{H}_{0}$ denote the crystalline Hamiltonian without the supplementary forces $\alpha_{i}$ and upper huts $\wedge$ represent operrators. The supplementary forces $\alpha_{i}$ are acted in the direction of the generalized coordinates $q_{i}$. The thermodynamic quantities of the anharmonic crystal (harmonic Hamiltonian) will be treated in the Einstein approximation.

After the action of the supplementary forces $p_{i}$ the system passes into a new equilibrium state. If the 0th atom in the lattice is affected by a supplementary force $p_{\alpha}$, then the total force acting on it must be zero, and one gets the force balance relation as

$$
\begin{aligned}
& \frac{1}{2} \sum_{i, \alpha}\left(\frac{\partial^{2} \varphi_{i 0}}{\partial u_{i \alpha} \partial u_{i \beta}}\right)_{e q}\left\langle u_{i \alpha}\right\rangle+\frac{1}{4} \sum_{i, \alpha, \gamma}\left(\frac{\partial^{3} \varphi_{i 0}}{\partial u_{i \alpha} \partial u_{i \beta} \partial u_{i \gamma}}\right)_{e q}\left\langle u_{i \alpha} u_{i \beta}\right\rangle \\
& +\frac{1}{12} \sum_{i, \alpha, \gamma, \eta}\left(\frac{\partial^{4} \varphi_{i 0}}{\partial u_{i \alpha} \partial u_{i \beta} \partial u_{i \gamma} \partial u_{i \eta}}\right)\left\langle u_{i \alpha} u_{i \gamma} u_{i \eta}\right\rangle-p_{\beta}=0
\end{aligned}
$$

The thermal averages of the atomic displacements $\left\langle u_{i \alpha} u_{i \gamma}\right\rangle$ and $\left\langle u_{i \alpha} u_{i \gamma} u_{i \eta}\right\rangle$ (called as second and third- order moments) at given site $\boldsymbol{R}_{i}$ can be expressed in terms of the first moment $\left\langle u_{i \alpha}\right\rangle$ with the aid of the recurence formula [12-14]. Then Equation (2) is transformed into the new differential equation:

$$
\begin{aligned}
& \gamma y^{3}+3 \theta \gamma y \frac{d y}{d p}+\gamma \theta^{2} \frac{d^{2} y}{d p^{2}}+\frac{\gamma \theta}{m \omega^{2}}(X-1) y \\
& +\beta y^{2}+\beta \theta \frac{d y}{d p}+\frac{\beta \theta}{m \omega^{2}}(X-1)+k y-p=0
\end{aligned}
$$

where $y \equiv\left\langle u_{j}\right\rangle_{P}, \quad X=x \operatorname{coth} x$, and $\theta=k_{B} T ; x=\frac{\hbar \omega}{2 \theta}$.

In the above Equation (3), $k, \gamma$ and $\beta$ are defined by

$$
\left.\begin{array}{l}
k=\frac{1}{2} \sum_{i}\left(\frac{\partial^{2} \varphi_{i 0}}{\partial u_{i \beta}^{2}}\right)_{e q} \equiv m \omega^{2} \\
\gamma=\frac{1}{12} \sum_{i}\left[\left(\frac{\partial^{4} \varphi_{i 0}}{\partial u_{i \beta}^{4}}\right)_{e q}+6\left(\frac{\partial^{4} \varphi_{i 0}}{\partial u_{i \beta}^{2} \partial u_{i \gamma}^{2}}\right)_{e q}\right] \\
\beta=\sum_{i}\left(\frac{\partial^{3} \varphi_{i 0}}{\partial u_{j x} \partial u_{j y} \partial u_{j z}}\right)_{e q}
\end{array}\right\}
$$

In deriving Equation (3) we have imposed the symmetry criterion for the thermal averages in the diamond $\mathrm{cu}-$ bic lattices as

$$
\left\langle u_{j \alpha}\right\rangle_{p}=\left\langle u_{j \beta}\right\rangle_{p}=\left\langle u_{j \gamma}\right\rangle_{p}=\left\langle u_{j}\right\rangle_{p} \equiv y
$$

Let us introduce the new variable y in the above Equation (3)

$$
y^{\prime}=y+\frac{\beta}{3 \gamma}
$$

Then we have the new differential equation instead of Equation (3)

$$
\begin{aligned}
& \gamma y^{\prime 3}+3 \theta \gamma y^{\prime} \frac{\mathrm{d} y^{\prime}}{\mathrm{d} p^{*}}+\gamma \theta^{2} \frac{\mathrm{d}^{2} y^{\prime}}{\mathrm{d} p^{* 2}}+\frac{\gamma \theta}{k}(X-1) y^{\prime} \\
& +K y^{\prime}-p^{*}=0
\end{aligned}
$$

where

$$
\begin{aligned}
& K=k-\frac{\beta^{2}}{3 \gamma} ; p^{*}=p-K^{*} ; \\
& K^{*}=\frac{\beta k}{\gamma}\left[\frac{2 \beta^{2}}{27 \gamma k}-\frac{1}{3}+\frac{2 \gamma \theta}{3 k^{2}}(X-1)\right]
\end{aligned}
$$

For higher temperatures, the relation xcothx $\sim 1$ holds and Equation (6) is reduced to

$$
\gamma \theta^{2} \frac{\mathrm{d}^{2} y^{\prime}}{\mathrm{d} p^{* 2}}+3 \theta \gamma y^{\prime} \frac{\mathrm{d} y^{\prime}}{\mathrm{d} p^{*}}+\gamma y^{\prime 3}+K y^{\prime}-p^{*}=0
$$


The nonlinear differential equation of Equation (8) can be solved in the following manner: We expand the solution $y^{\prime}$ in terms of the "force" $p^{*}$ up to the second order as

$$
y^{\prime}=y_{0}^{\prime}+A_{1} p^{*}+A_{2} p^{* 2}
$$

where $A_{1}$ and $A_{2}$ are the constants [12]. The above Equation (8) is solved as

$$
y_{0}^{\prime} \approx \sqrt{\frac{2 \gamma \theta^{2}}{3 K^{3}} A}
$$

with

$$
\begin{aligned}
A= & a_{1}+\frac{\gamma^{2} \theta^{2}}{K^{4}} a_{2}+\frac{\gamma^{3} \theta^{3}}{K^{6}} a_{3}+\frac{\gamma^{4} \theta^{4}}{K^{8}} a_{4} \\
& +\frac{\gamma^{5} \theta^{5}}{K^{10}} a_{5}+\frac{\gamma^{6} \theta^{6}}{K^{12}} a_{6}
\end{aligned}
$$

and $a_{1}=3 / 2 ; a_{2}=33 / 2 ; a_{3}=-51 ; a_{4}=458 / 3$;

$a_{5}=-1589 / 3 ; a_{6}=1633$.

Here, $y_{0}^{\prime}$ represents the atomic displacement for the case when the force $p^{*}$ is zero. The general solution of Equation (3) is solved as

$$
\begin{aligned}
y_{0} & =\left.y\right|_{p=0}=\left.y^{\prime}\right|_{p^{*}=-K^{*}}-\frac{\beta}{3 \gamma} \\
& =y_{0}^{\prime}-\frac{\beta}{3 \gamma}+\frac{1}{K}\left(1+\frac{6 \gamma^{2} \theta^{2}}{K^{4}}\right)\left[\frac{1}{3}-\frac{2 \beta^{2}}{27 \gamma k}\right] \frac{\beta k}{\gamma}
\end{aligned}
$$

Once the thermal expansion $y_{0}$ of the lattice is found, one can get the Helmholtz free energy of the system in the following form

$$
\psi=U_{0}+\psi_{0}+\psi_{1}
$$

where $\psi_{0}$ denotes the free energy in the harmonic approximation and $\psi_{1}$ the anharmonic contribution to the free energy. The Helmholtz free energy of our system can be derived from the Hamiltonian $\mathrm{H}$ of the following form:

$$
\widehat{H}=\widehat{H}_{0}-\lambda \widehat{V}
$$

where $\widehat{H}_{0}$ denote the Hamiltonian of the harmonic approximation, $\lambda$ the parameter and $\vec{V}$ the anharmonic vibrational contributions. Following exactly the general formular in the SMM formulation [12], one can get the free energy $\psi$ of the system as

$$
\psi=U_{0}+\psi_{0}-\int_{0}^{\lambda}\langle\hat{V}\rangle_{\lambda} \mathrm{d} \lambda
$$

where $\lambda \hat{V}$ represents the Hamiltonian corresponding $t$ o the anharmonicity contribution. Then the free energy of the system is given by

$$
\begin{aligned}
\psi \approx & U_{0}+\psi_{0}+\frac{3 N \theta^{2}}{k^{2}}\left\{\gamma_{2} X^{2}-\frac{2 \gamma_{1}}{3}\left(1+\frac{X}{2}\right)\right\}+3 N \frac{2 \theta^{3}}{k^{4}} \\
& \cdot\left\{\frac{4}{3} \gamma_{2}^{2} X\left(1+\frac{X}{2}\right)-2\left(\gamma_{1}^{2}+2 \gamma_{1} \gamma_{2}\right)\left(1+\frac{X}{2}\right)(1+X)\right\} \\
& +3 N M(k-K)\left(\frac{-\beta^{3} k}{27 \gamma^{3}}\right)+3 N \theta M \\
& \cdot\left\{\left[\frac{3}{K}\left(\frac{k}{K}-1\right)+\left(\frac{1}{K}-\frac{1}{k}\right)(X-1)\right]\left(\frac{-\beta^{3} k}{27 \gamma^{2}}\right)-\frac{K \beta}{3 \gamma}\right\} \\
& +3 N \theta\left[M^{2}\left(\frac{-\beta^{2} k K}{6 \gamma^{2}}\right)\right] \\
& +3 N \theta\left[\left(\frac{k}{K^{2}}-\frac{1}{K}\right)+\left(\frac{1}{3 K}-\frac{1}{3 k}\right)(X-1)\right]\left(\frac{\beta^{2}}{6 \gamma}\right) \\
& +3 N \theta^{2}\left\{M^{3}\left(\frac{k}{K}-1\right)\left(\frac{-\beta^{3} k}{27 \gamma^{2}}\right)+M\left(\frac{-2 \beta^{3} k^{2}}{9 K^{4} \gamma}\right)\right\} \\
& +3 N \theta^{2} \frac{2}{K^{3}} a_{1}\left[\left(\frac{3}{K}+\frac{1}{k}(X-1)\right)\right]\left(\frac{-\beta^{2} k}{18}\right) \\
+ & 3 N \theta^{2} M^{2}\left(\frac{k}{K}-1\right)\left(\frac{\beta^{2}}{6 \gamma}\right)
\end{aligned}
$$

where $M=\left(\frac{2 \gamma a_{1}}{3 K^{3}}\right)^{\frac{1}{2}}$, and the second term of above Equation (15) denotes the harmonic contribution to the free energy

$$
\psi_{0}=3 N \theta\left[x+\ln \left(1-\mathrm{e}^{-2 x}\right)\right]
$$

with the aid of the "real space" free energy formula $\psi=E-T S$, one can find the thermodynamic quantities of given systems. The thermodynamic quantities such as specific heats and elastic modul at temperature $T$ are directly derived from the free energy $\psi$ of the system.

\subsection{Thermodynamic Properties of Semiconductors with Defects}

The Gibbs free energy of crystals consisting of $N$ atoms and $n \ll N$ vacancies has the form:

$$
G(T, P)=G_{0}(T, P)+n g_{V}^{f}-T S_{C}
$$

where $G_{0}(T, P)$ is the Gibbs free energy of the perfect crystals consisting $\mathrm{N}$ atoms, $g_{V}^{f}(T, P)$ is the Gibbs energy change on forming a single vacancy, $S_{C}$-the entropy of mixing:

$$
S_{C}=k_{B} \ln \frac{(N+n) !}{N ! n !}
$$


From the minimization condition of the Gibbs free ene rgy of the crystal with point defects, we obtain the eq uilibrium concentration of the vacancies as $[15,16]$

$$
n_{v}=\exp \left\{-\frac{g_{v}^{f}(P, T)}{\theta}\right\}
$$

where $g_{v}^{f}$ is the change in the Gibbs free energy due to the fomation of a vacancy and can be given by

$$
g_{V}^{f}(T, P)=-u_{0}+\Delta \psi_{0}^{*}+P \Delta V
$$

It should be noted that pressure affects the diffusivity through both the free energies, $\psi_{0}^{*}$, and the volume change, resulting from the formation of the point defect, $\Delta V$. This change is due to the $P \Delta V$ work done by the pressure medium against the volume change associated with defect formation and migration.

In the above Equation (20),

$u_{0}=\frac{1}{2} \sum_{i} \varphi_{i 0}\left(\boldsymbol{r}_{i} \mid\right)+\frac{1}{3} \sum_{i, j} W_{i j o}\left(r_{i}, r_{j}, r_{o}\right)$ represent the internal energy associated with atom 0 and $\varphi_{i o}$ the effective interaction energies between the $o^{\text {th }}$ and $i^{\text {th }}$ atoms, $\Delta \psi_{o}^{*}$ denotes the change in the Helmholtz free energy of the central atom which creates the vacancy, by moving itself to a certain sink site in the crystal, and is given by

$$
\Delta \psi_{o}^{*}=(C-1) \Delta \psi_{o}^{*}
$$

where $\psi_{0}^{*}$ denotes the free energy of the central atom after moving to a certain sink sites in the crystal, $C$ is simply regarded as a numerical factor. In the previous paper [17], we take the average value for $C$ as

$$
C \approx 1+\frac{u_{o}}{2 \Delta \psi_{o}^{*}}
$$

From Equations (20), (21), and (22) we obtain the expression of the Gibbs energy change on forming a single vacancy:

$$
g_{V}^{f}(T, P) \approx-\frac{u_{0}}{2}+P \Delta V
$$

Form Equations (17) and (23) it is easy to obtain the expression for the Helmholtz free energy of crystals with defects:

$$
\psi_{\text {def }}=\psi-n N \cdot \frac{u_{0}}{2}-T S_{C}
$$

Applying the Gibbs-Helmholtz relation and using Equation (24) we find the expression for the energy of a crystal with defects and so the specific heat at constant volume $C_{V}$ has the form

$$
\begin{aligned}
C_{V}^{\text {def }}= & C_{V}+\frac{N n_{V}}{2} \\
& \cdot\left\{\frac{1}{T}\left(g_{V}^{f}-\theta \frac{\partial g_{V}^{f}}{\partial \theta}\right)\left[T \frac{\partial u_{0}}{\partial T}-u_{0}\left(\frac{\partial g_{V}^{f}}{\partial \theta}-\frac{g_{V}^{f}}{\theta}+1\right)\right]\right. \\
& +T \frac{\partial^{2} u_{0}}{\partial T^{2}}-\frac{\partial u_{0}}{\partial T}\left(\frac{\partial g_{V}^{f}}{\partial \theta}-\frac{g_{V}^{f}}{\theta}\right) \\
& \left.-u_{0} k_{B}\left(\frac{\partial^{2} g_{V}^{f}}{\partial \theta^{2}}-\frac{\theta \frac{\partial g_{V}^{f}}{\partial \theta}-g_{V}^{f}}{\theta^{2}}\right)\right\}
\end{aligned}
$$

In the case of zero pressure, $g_{V}^{f}(T, 0) \approx-\frac{u_{0}}{2}$, the specific heat at constant volume $C_{V}^{\text {def }}$ of crystal with defects has the simple form:

$$
\begin{aligned}
C_{V}^{\text {def }}= & C_{V}+\frac{N n_{V} k_{B}}{2} \\
& \cdot\left\{\frac{1}{2 \theta^{2}}\left(-u_{0}+\theta \frac{\partial u_{0}}{\partial \theta}\right)\left[\theta \frac{\partial u_{0}}{\partial \theta}+u_{0}\left(\frac{\partial u_{0}}{2 \partial \theta}-\frac{u_{0}}{2 \theta}-1\right)\right]\right. \\
& +\theta \frac{\partial^{2} u_{0}}{\partial \theta^{2}}+\frac{\partial u_{0}}{\partial \theta}\left(\frac{\partial u_{0}}{2 \partial \theta}-\frac{u_{0}}{\theta}\right) \\
& \left.+\frac{u_{0}}{2}\left(\frac{\partial^{2} u_{0}}{\partial \theta^{2}}-\frac{1}{\theta} \frac{\partial u_{0}}{\partial \theta}+\frac{u_{0}}{2 \theta^{2}}\right)\right\}
\end{aligned}
$$

where $C_{V}$ is the specific heat at constant volume of perfect crystal [10].

The equation of states of the system with defects at finite temperature $T$ is now obtained from Equation (24) and the pressure $P$ of the system is given by the derivative of the free energy with respect to volume as

$$
P=-\left(\frac{\partial \psi_{\text {def }}}{\partial V}\right)_{T}=-\frac{a}{3 V}\left(\frac{\partial \psi_{\text {def }}}{\partial a}\right)_{T}
$$

or

$$
P . v=-\frac{a}{3 N}\left(\frac{\partial \psi_{\text {def }}}{\partial a}\right)_{T}
$$

where $v$ is the atomic volume.

From the Equations (24) and (27) one can find the equation of states of the crystal with defects at zero pressure in the harmonic approximation 


$$
\left(\frac{\partial \psi}{\partial a}\right)_{T}=\frac{N n_{V}}{2}\left(\frac{\partial u_{0}}{\partial a}\right)_{T}\left[1+\frac{u_{0}}{2 \theta}\right]
$$

From the Equation (28) one can find the average nearest-neighbor distance (NND), $a(0, T)$ of atoms in crystal at zero pressure and temperature T. Equation (28) can be solved using a computational program to find out the values of the NND of the crystals with defects, $a(0, T)$.

Let us now consider the compressibility of the solid phase (diamond and zinc-blende structures). The isothermal compressibility can be given as

$$
\chi_{T}=-\frac{1}{V_{o}}\left(\frac{\partial V}{\partial P}\right)_{T}=\frac{3\left(\frac{a(P, T)}{a(P, 0)}\right)^{3}}{2 P+\frac{a^{2}(P, T)}{3 V(P, T)}\left(\frac{\partial^{2} \Psi_{d e f}}{\partial a^{2}}\right)_{T}}
$$

where

$$
\begin{aligned}
& \left(\frac{\partial^{2} \psi_{\text {def }}}{\partial a^{2}}\right)_{T}=\left(\frac{\partial^{2} \psi}{\partial a^{2}}\right)_{T}-\frac{N n_{V}}{2}\left(\frac{\partial^{2} u_{0}}{\partial a^{2}}\right)_{T}\left(1+\frac{u_{0}}{2 \theta}\right) \\
& +\frac{N n_{V}}{2}\left(\frac{\partial u_{0}}{\partial a}\right)_{T}^{2}\left(1+\frac{u_{0}}{4 \theta}\right) \frac{1}{\theta}
\end{aligned}
$$

In the case of zero pressure, the expression of the isothermal compressibility for crystals with defects is given as

$$
\chi_{T}^{\text {def }}=
$$

$$
\frac{3\left(\frac{a}{a_{0}}\right)^{3}}{\frac{a^{2}}{3 V}\left(\frac{\partial^{2} \psi}{\partial a^{2}}\right)_{T}}
$$

$$
\left\{1-\frac{N n_{V}}{2\left(\frac{\partial^{2} \psi}{\partial a^{2}}\right)_{T}}\left[\left(\frac{\partial^{2} u_{0}}{\partial a^{2}}\right)_{T}\left(1+\frac{u_{0}}{2 \theta}\right)+\left(\frac{\partial u_{0}}{\partial a}\right)_{T}^{2}\left(1+\frac{u_{0}}{4 \theta}\right) \frac{1}{\theta}\right]\right\}
$$

or

$$
\frac{\chi_{T}^{\text {def }}=}{1-\frac{N n_{V}}{2\left(\frac{\partial^{2} \psi}{\partial a^{2}}\right)_{T}}\left[\left(\frac{\partial^{2} u_{0}}{\partial a^{2}}\right)_{T}\left(1+\frac{u_{0}}{2 \theta}\right)+\left(\frac{\partial u_{0}}{\partial a}\right)_{T}^{2}\left(1+\frac{u_{0}}{4 \theta}\right) \frac{1}{\theta}\right]}
$$

with $\chi_{T}$ is the isothermal compressibility of perfective crystals at zero pressure

$$
\chi_{T}=\frac{3\left(\frac{a}{a_{0}}\right)^{3}}{\frac{a^{2}}{3 V}\left(\frac{\partial^{2} \psi}{\partial a^{2}}\right)_{T}}
$$

The specific heat at constant pressure, $C_{P}$ of crystal with defects is determined from the well known thermodynamic relations

$$
C_{P}^{\text {def }}=C_{V}^{\text {def }}+\frac{9 T V}{\chi_{T}^{\text {def }}}\left(\alpha^{\text {def }}\right)^{2}
$$

where the thermal expansion coefficient $\alpha^{\text {def }}$ of defective crystal is given as

$$
\alpha^{\text {def }}=-\frac{k_{B} \chi_{T}^{\text {def }}}{3}\left(\frac{a_{0}}{a}\right)^{2} \frac{a}{3 V} \frac{\partial^{2} \psi_{\text {def }}}{\partial \theta \partial a}
$$

\section{Results and Discussion}

To calculate the thermodynamic quantities of $\mathrm{Si}$ and GaAs crystals with defects, we will use the many-body potential [18], which include both the two-body and the three-body atomic interactions

$$
\begin{aligned}
& \Phi=\sum_{i, j} \varphi_{i j}+\sum_{i, j, k} W_{i j k}, \\
& \varphi_{i j}=\varepsilon\left[\left(\frac{r_{0}}{r_{i j}}\right)^{12}-2\left(\frac{r_{0}}{r_{i j}}\right)^{6}\right], \\
& W_{i j k}=Z \frac{\left(1+3 \cos \theta_{i} \cos \theta_{j} \cos \theta_{k}\right)}{\left(r_{i j} r_{j k} r_{k i}\right)^{3}} .
\end{aligned}
$$

The parameters were fitted to the bond lengths of the dimer and trimer and the lattice parameters and cohesive energy of the diamond structure. Parameters of the manybody potential for monoatomic (A), binary (A-B) systems are given in Tables $\mathbf{1}$ and $\mathbf{2}$, respectively.

In Table 3, we compare the calculation results of the specific heats at constant pressure, $C_{P}$ of Si crystal with defects obtained by using the SMM analytic formula with the experimental results of Ref. [19]. Here, it should be noted that the equilibrium concentration of the vacancies is very small at low temperature. At high temperature being near the melting one the contribution of the vacan-

Table 1. Parameters of many body potential $r_{0}, Z$, and $\varepsilon$ for Si [18].

\begin{tabular}{ll}
\hline Quantity & Si \\
\hline $\boldsymbol{\varepsilon}_{\mathrm{AA}}(\mathbf{e V})$ & 2.81 \\
$\boldsymbol{r}_{\mathbf{0 A A}}\left(\mathbf{A}^{\mathbf{0}}\right)$ & 2.295 \\
$\boldsymbol{Z}_{\mathrm{AAA}}\left(\mathbf{e V A}^{\mathbf{0 9}}\right)$ & 3484.0 \\
\hline
\end{tabular}


Table 2. Parameters of many body potential $r_{0}, Z$, and $\varepsilon$ for GaAs [18].

\begin{tabular}{ll}
\hline Quantity & GaAs \\
\hline$\varepsilon_{\mathrm{AA}}(\mathbf{e V})$ & 1.738 \\
$\boldsymbol{r}_{0 \mathrm{AA}}\left(\mathbf{A}^{\mathbf{0}}\right)$ & 2.448 \\
$\boldsymbol{Z}_{\mathrm{AAB}}\left(\mathbf{e V A}^{09}\right)$ & 1900.0 \\
$\mathbf{Z}_{\mathrm{ABB}}\left(\mathbf{e V}^{\mathbf{0}} \mathbf{A}^{\mathbf{9}}\right)$ & 4600.0 \\
$\mathbf{Z}_{\mathrm{AAA}}\left(\mathbf{e V}^{\mathbf{0}}\left(\mathbf{A}^{\mathbf{9}}\right)^{\mathbf{9}}\right)$ & 1826.4 \\
\hline
\end{tabular}

cies on the specific heats at constant volume, $C_{V}$ and those at constant pressure $C_{P}$ of $\mathrm{Si}$ crystal is about $0.5 \%$. In Table 3, we also present the SMM calculations of the specific heats at constant volume, $C_{V}$ and $\Delta C_{V}=C_{V}^{\text {def }}-C_{V}$ for Si crystal with defects.

In Table 4, we present the SMM results of the equilibrium concentration of the vacancies, the specific heats at constant volume, $C_{V}$ and $\Delta C_{V}=C_{V}^{\text {def }}-C_{V}$, and compare the calculation results of the specific heats at constant pressure, $C_{P}$ for GaAs defective crystal with the experimental results [20].

The linear thermal expansion coefficient of Si crystal is calculated using the many-body potential. The Figure 1 shown that the many-body potential gives reasonable values of thermal expansion coefficient compared with the experimental results [19]. In Figures 2 and 3, we present the temperature dependence of the specific heat at constant pressure $C_{P}$ of $\mathrm{Si}$ and GaAs crystals with defects, by dashed lines, in comparison with the corresponding experimental results $[19,20]$, by solid lines. Figures 2 and 3 show the SMM specific heats at constant pressure, $C_{P}$ (dashed lines) of the diamond cubic Si and zincblende GaAs crystals with defects, calculated by using

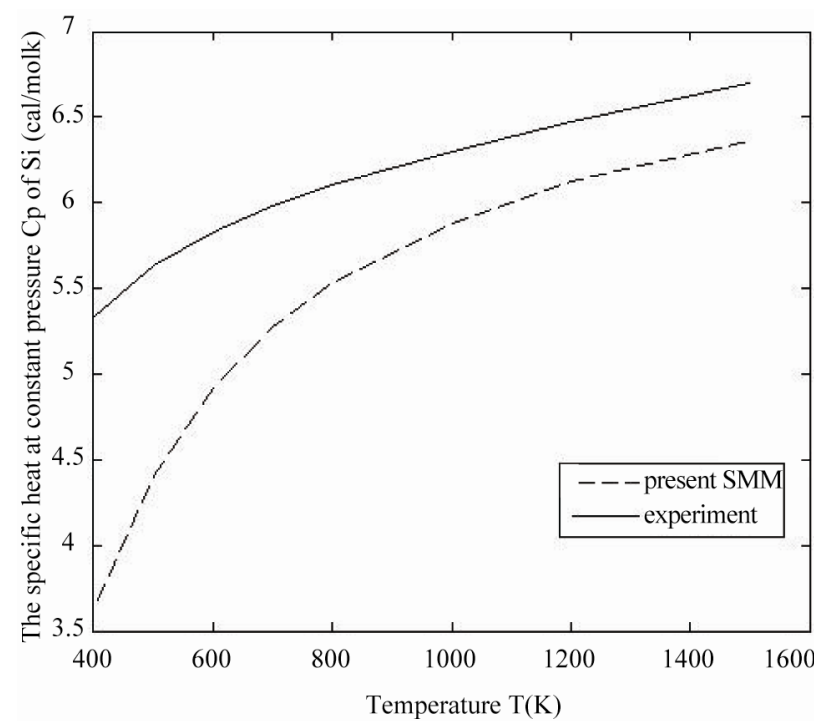

Figure 1. Temperature dependence of the linear thermal expansion coefficient of Si crystal with defects.

the many-body potentials, as a function of the temperature. One can see in Figures $\mathbf{2}$ and $\mathbf{3}$ that the specific heats at constant pressure, $C_{P}$ increase with the temperature, in agreement with the experimental results $[19,20]$.

\section{Conclusions}

The thermodynamic properties of semiconductors with defects have been studied using statistical moment method. We have presented the SMM formulation for the thermodynamic quantities of diamond cubic and zincblende semiconductors with defects taking into account

Table 3. SMM calculated temperature dependence of thermodynamic quantities for Si crystal with defects.

\begin{tabular}{llllllllllllll}
\hline $\mathrm{T}(\mathrm{K})$ & 300 & 400 & 500 & 600 & 700 & 800 & 900 & 1000 & 1100 & 1200 & 1300 & 1400 & 1500 \\
\hline$n_{v}$ & 6.3 & 6.8 & 4.5 & 7.3 & 1.5 & 7.7 & 1.7 & 2.0 & 1.5 & 8.1 & 3.4 & 1.2 & 3.3 \\
& $10^{-38}$ & $10^{-25}$ & $10^{-20}$ & $10^{-17}$ & $10^{-14}$ & $10^{-13}$ & $10^{-11}$ & $10^{-10}$ & $10^{-9}$ & $10^{-9}$ & $10^{-8}$ & $10^{-7}$ & $10^{-7}$ \\
$-\Delta C_{V}$ & 7.1 & 3.2 & 1.1 & 1.0 & 1.3 & 4.5 & 6.9 & 5.9 & 3.3 & 1.4 & 4.5 & 1.2 & 2.8 \\
& $10^{-26}$ & $10^{-18}$ & $10^{-13}$ & $10^{-10}$ & $10^{-8}$ & $10^{-7}$ & $10^{-6}$ & $10^{-5}$ & $10^{-4}$ & $10^{-3}$ & $10^{-3}$ & $10^{-2}$ & $10^{-2}$ \\
$C_{V}^{\text {def }}$ & 2.369 & 3.577 & 4.318 & 4.793 & 5.107 & 5.326 & 5.484 & 5.603 & 5.696 & 5.768 & 5.826 & 5.868 & 5.895 \\
$C_{P}^{\text {def }}$ & 2.39 & 3.63 & 4.41 & 4.92 & 5.28 & 5.53 & 5.73 & 5.88 & 6.01 & 6.12 & 6.21 & 6.30 & 6.36 \\
$C_{P}$ exp.[16] & - & 5.33 & 5.63 & 5.83 & 5.98 & 6.10 & - & 6.30 & - & 6.47 & - & - & 6.70 \\
\hline
\end{tabular}

Table 4. SMM calculated temperature dependence of thermodynamic quantities for GaAs crystal with defects.

\begin{tabular}{llllllllll}
\hline $\mathrm{T}(\mathrm{K})$ & 300 & 400 & 500 & 600 & 700 & 800 & 900 & 1000 & 1200 \\
\hline$n_{v}$ & 1.5 & 7.6 & 2.0 & 3.9 & 1.7 & 2.8 & 2.5 & 1.4 & 2.0 \\
& $10^{-23}$ & $10^{-18}$ & $10^{-14}$ & $10^{-12}$ & $10^{-10}$ & $10^{-9}$ & $10^{-8}$ & $10^{-7}$ & $10^{-6}$ \\
$-\Delta C_{V}$ & 6.0 & 1.3 & 1.7 & 1.9 & 5.1 & 5.7 & 3.6 & 1.5 & 12.1 \\
& $10^{-17}$ & $10^{-11}$ & $10^{-8}$ & $10^{-6}$ & $10^{-5}$ & $10^{-4}$ & $10^{-3}$ & $10^{-2}$ & $10^{-2}$ \\
$C_{P}^{\text {def }}$ & 5.22 & 5.67 & 5.93 & 6.11 & 6.24 & 6.36 & 6.48 & 6.55 & 6.76 \\
$C_{P}$ exp.[16] & 5.65 & 5.79 & 5.93 & 6.07 & 6.21 & 6.35 & 6.49 & 6.63 & 6.91 \\
\hline
\end{tabular}




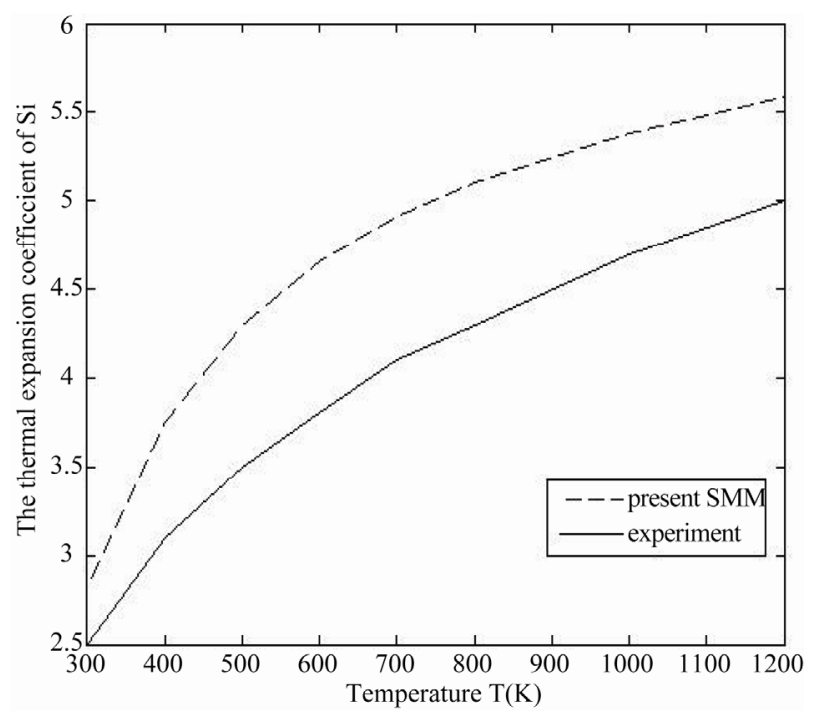

Figure 2. Temperature dependence of the specific heat at constant pressure $C_{P}$ of $\mathrm{Si}$ crystal with defects.

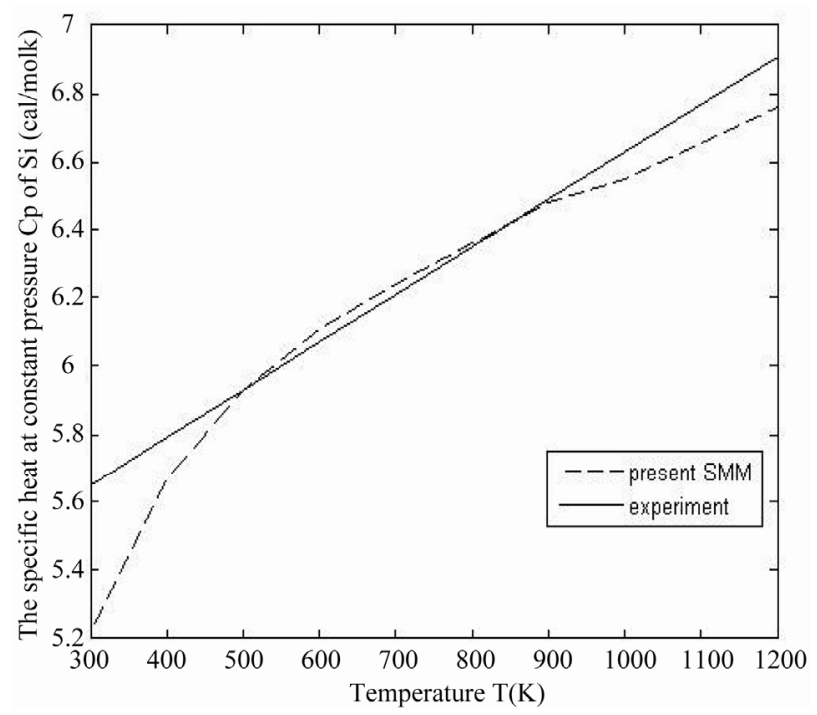

Figure 3. Temperature dependence of the specific heat at constant pressure $C_{P}$ of GaAs crystal with defects.

the higher order (fourth order) anharmonic vibrational terms in the Helholtz free energy and derived the various thermodynamic quantities in closed analytic forms. The lattice constants, linear thermal expansion coefficients, specific heats at constant volume and those at constant pressure, $C_{V}$ and $C_{P}$, have been calculated successfully for the Si and GaAs crystals with defects. We have calculated thermodynamic quantities for Si and GaAs semiconductors with defects and these calculated SMM thermodynamic quantities are in good agreement with previous experiments. The temperature dependence of the thermodynamic quantities of $\mathrm{Si}$ and GaAs semiconductors with defects being estimated.

\section{Acknowledgments}

This work is supported by the research project No. 103.01.2609 of NAFOSTED.

\section{REFERENCES}

[1] D. Bimberg, M. Grundman, and N. N. Ledentsov, "Quantum Dot Heterostructures," Wiley, New York, 2001.

[2] A. L. Efros, D. J. Lockwood and L. Tsybeskov (Eds.), "Semiconductor Nanocrystals, from Basic Principles to Applications,” Klwer-Acadimic/Plenum, New York, 2003.

[3] K. Chung and J. B. Xia, "Spatially Separated Excitons in Quantum-Dot Quantum Well Structures” Physical Review $B$, Vol. 57, No. 16, 1998, pp. 9780-9786. doi:10.1103/PhysRevB.57.9780

[4] X. R. Qin, B. S. Swartzentruber and M. G. Lagally, "Scanning Tunneling Microscopy Identification of AtomicScale Intermixing on Si(100) at Submonolayer Ge Coverages,” Physical Review Letters, Vol. 84, No. 20, 2000, pp. 4645-4648. doi:10.1103/PhysRevLett.84.4645

[5] M. T. Yin and M. L. Cohen, "Theory of Lattice-Dynamical Properties of Solids: Application to Si and Ge," Physical Review B, Vol. 26, No. 6, 1982, pp. 3259-3272. doi:10.1103/PhysRevB.26.3259

[6] M. T. Yin, and M. L. Cohen, "Theory of Static Structural Properties, Crystal Stability, and Phase Transformations: Application to Si and Ge,” Physical Review B, Vol. 26, No. 10, 1982, pp. 5668-5687. doi:10.1103/PhysRevB.26.5668

[7] M. T. Yin, and M. L. Cohen, "Theory of Ab Initio Pseudopotential Calculations,” Physical Review B, Vol. 25, No. 12, 1982, pp. 7403-7412. doi:10.1103/PhysRevB.25.7403

[8] O. Sugino, and R. Car, “Ab Initio Molecular Dynamics Study of First-Order Phase Transitions: Melting of Silicon,” Physical Review Letters, Vol. 74, No. 10, 1995, pp. 1823-1826. doi:10.1103/PhysRevLett.74.1823

[9] P. Focher, G. L. Chiarotti, M. Bernasconi, E. Tosatti and M. Parrimello, "Structural Phase Transformations via FirstPrinciples Simulation,” Europhysics Letters, Vol. 26, No. 5, 1994, pp. 345-351. doi:10.1209/0295-5075/26/5/005

[10] V. V. Hung, K. Masuda-Jindo and P. T. M. Hanh, “Application of the Statistical Moment Method to Thermodynamic Quantities of Silicon,” Journal of Physics: Condensed Matter, Vol. 18, No. 1, 2006, pp. 283-293. doi:10.1088/0953-8984/18/1/021

[11] V. V. Hung, K. Masuda-Jindo, P. T. M. Hanh and N. T. Hai, "Equation of States and Melting Temperatures of Diamond Cubic and Zinc-Blende Semiconductors: Pressure Dependence,” Journal of Physics: Conference Series, Vol. 98, 2008, pp. 032001-032006.

[12] N. Tang and V. V. Hung, "Investigation of the Thermodynamic Properties of Anharmonic Crystals by the Moment Method: I. General Results for Face-Centred Cubic Crystals,” Physica Status Solidi B, Vol. 149, No. 2, 1988, 
pp. 511-519. doi:10.1002/pssb.2221490212

[13] N. Tang and V. V. Hung, "Investigation of the Thermodynamic Properties of Anharmonic Crystals by the Moment Method: III. Thermodynamic Properties of the Crystals at Various Pressures,” Physica Status Solidi (B), Vol. 162, No. 2, 1990, pp. 371-377. doi:10.1002/pssb.2221620206

[14] K. Masuda-Jindo, V. V. Hung and P. D. Tam, “Thermodynamic Quantities of Metals Investigated by an Analytic Statistical Moment Method,” Physical Review B, Vol. 67, 2003, pp. 094301-094315. doi:10.1103/PhysRevB.67.094301

[15] V. V. Hung, N. T. Hai and N. Q. Bau, "Investigation of the Thermodynamic Properties of Anharmonic Crystals with Defects by the Moment Method," Journal of the Physical Society of Japan, Vol. 66, No. 11, 1997, pp. 34943498. doi:10.1143/JPSJ.66.3494

[16] V. V. Hung, J. Lee, K. Masuda-Jindo and P. T. T. Hong,
"Study of Self-Diffusion in Silicon at High Pressure," Journal of the Physical Society of Japan, Vol. 75, No. 2, 2006, pp. 024601-024608. doi:10.1143/JPSJ.75.024601

[17] V. V. Hung, P. T. T. Hong and N. T. Hai, "Study of SelfDiffusion in GaAs Crystal: Temperature Dependence,” Communications in Physics, Vol. 20, No. 3, 2010, pp. 227-231.

[18] S. Erkoc, "Empirical Many-Body Potential Energy Functions Used in Computer Simulations of Condensed Matter Properties,” Physics Reports, Vol. 278, No. 2, 1997, pp. 79-105. doi:10.1016/S0370-1573(96)00031-2

[19] D. E. Gray, “American Institute of Physics Handbook," Second Edition, McGraw-Hill, New York, 1963, pp. 466-451.

[20] M. P. Shaskolskoi, “Acoustic Crystals,” Science, Moscow, 1982. 